\title{
Chlamydia and Gonorrhea Screening in San Francisco High Schools
}

\author{
CHARLOTTE K. KENT, MPH, ${ }^{*}$ ANNA BRANZUELA, BA, ${ }^{*}$ LYN FISCHER, MPA, * \\ TRISH BASCOM, RN, BSN, MA, ${ }^{\dagger}$ AND JEFFREY D. KLAUSNER, MD, MPH*
}

Background: Previous school-based studies in cities with a high prevalence of chlamydia found a substantial prevalence of chlamydial infection among students.

Goal: The goal was to determine the feasibility and acceptability of chlamydia and gonorrhea screening in San Francisco high schools.

Study Design: Sexually transmitted disease (STD) education and screening were conducted at four high schools. Students provided basic demographic information and urine specimens for chlamydia and gonorrhea ligase chain reaction testing.

Results: Among 283 asymptomatic females screened, 3.9\% had chlamydia and $0.7 \%$ had gonorrhea. The prevalence of chlamydia was $1.5 \%$ among females $<16$ years of age and $4.6 \%$ among females $\geq 16$ years of age. Only $0.8 \%$ of asymptomatic males (3/381) had chlamydia, and none had gonorrhea.

Conclusion: STD screening was both feasible and acceptable in San Francisco high schools. STD screening in high schools should be prioritized as follows: (1) chlamydia screening over gonorrhea screening, (2) female screening over male screening, and (3) screening of older students (juniors and seniors) over screening of younger students.

CHLAMYDIA AND GONORRHEA are primarily asymptomatic infections that are important causes of pelvic inflammatory disease, tubal infertility, and ectopic pregnancy, and rates of both are consistently highest among females younger than 20 years of age. ${ }^{1}$ In light of this, performing chlamydia screening in high schools is a strategy to target adolescents at greatest risk for chlamydia, who may not otherwise access or be provided with sexually transmitted disease (STD) screening services.

Previous school-based studies in cities with a high prev-

The authors thank Sally Liska and the staff of the Public Health Laboratory; Jerome Johnson, Gloria Calero, and disease control investigators and health workers from STD Services; Roni Robertson and the staff at the Balboa Teen Clinic; the staff of the San Francisco Unified School District Health Programs; and the health teachers and students at the participating schools.

Reprint requests: Charlotte Kent, MPH, STD Prevention and Control Services, 1360 Mission Street, Suite 401, San Francisco, CA 94103. E-mail: Charlotte_Kent@dph.sf.ca.us.

Received for publication June 16, 2001, revised October 24, 2001, and accepted November 2, 2001.
From *STD Prevention and Control Services, Department of Public Health, and ${ }^{\dagger}$ School Health Programs Department, Unified School District, San Francisco,

California

alence of chlamydia revealed a substantial prevalence of chlamydial infection among students: $11 \%$ among sexually active female Baltimore students ${ }^{2,3}$ and $12 \%$ and $6 \%$ among female and male students, respectively, in New Orleans., 4 In Seattle, a city with moderate rates of chlamydia, $6 \%$ of students screened at school-based clinics had chlamydia. ${ }^{6}$ We piloted screening for chlamydia and gonorrhea in high schools in San Francisco, a city with a moderate prevalence of chlamydia and gonorrhea, to determine the feasibility and acceptability of screening in this venue.

\section{Methods}

San Francisco Department of Public Health, STD Prevention and Control Services, in conjunction with the San Francisco Unified School District, School Health Programs Department, performed STD education and screening in four of the 19 high schools in San Francisco during the April 1999 "STD Awareness Month." Presentations at a school board meeting by the Director of STD Services, a teen clinic practitioner, and the coordinator of the school screenings provided information about the STD education and screening program to the community and especially parents. The school board unanimously supported the STD project resolution.

STD screening was open to all students, regardless of sex, age, or history of sexual activity. STD education was provided in classrooms or individually before screening. Parents of students at each participating school were informed via letter about the STD education and screening program and could waive their child's participation in the educational component. Parental consent is not required for STD screening or contraceptive services in California for persons aged 12 years or older. 
TABLE 1. STD Service Staff Time and Proportion of High School Students Screened: San Francisco, 1999

\begin{tabular}{ccllrlrr}
\hline High School & $\begin{array}{c}\text { Total } \\
\text { Students }\end{array}$ & $\begin{array}{c}\text { Screening } \\
\text { Setting }\end{array}$ & \multicolumn{1}{c}{ Time } & $\begin{array}{c}\text { STD Staff } \\
\text { Hours }\end{array}$ & $\begin{array}{c}\text { N Screened of Total } \\
\text { Students } \\
\text { Screened }\end{array}$ & $\begin{array}{c}\text { N/Staff } \\
\text { Hour }\end{array}$ \\
\hline A & 1228 & School clinic & Clinic hours & 72 & 281 & 3.9 & $22.9 \%$ \\
B & 2457 & Health fair & 10 AM to 12 PM & 14 & 151 & 10.8 & $6.2 \%$ \\
C & 632 & Small room & Nonschool hours & 16 & 136 & $21.5 \%$ \\
D & 180 & Lunchroom & School hours & 36 & 96 & 2.7 & $53.3 \%$ \\
Total & 4497 & Varied & Varied & 138 & 664 & 4.8 \\
\hline
\end{tabular}

${ }^{\star} \mathrm{A}$ and $\mathrm{B}=$ comprehensive; $\mathrm{C}=$ preparatory; $\mathrm{D}=$ continuation.

STD $=$ sexually transmitted disease.

The School Health Programs staff reviewed and assisted in the revision of the STD Service's health education materials, to tailor them more specifically to their student population. The School Health Programs staff also selected the four pilot schools and ensured that they included a variety of types of high schools. Two of the participating schools were comprehensive high schools (schools A and B). Comprehensive high schools are large, with a broad but relatively traditional curriculum. School C was a small, college preparatory school where admission is through lottery. School D was a continuation school for students at high risk for dropping out or in need of extra attention.

The screening coordinator met with each school principal or school-designated health liaison to coordinate and develop a campaign to maximize student participation. The setting (health fair, school-based clinic, lunchroom) and times for screening varied by school. In school D, individual students could not be released from class to be screened; instead, entire classrooms were released for screening.

STD Services staff members provided all health education and confidential screening. To increase interest in screening, STD Services provides incentives during many community outreach activities. For the high school screening, participating students selected gift certificates valued up to $\$ 10$. Only demographic information was collected from screened students.

Ligase chain reaction assays (LCx; Abbott Laboratories) for chlamydia and gonorrhea were performed on urine specimens by the Department of Public Health Laboratory. Students whose test was positive were counseled and treated with a directly observed single-dose therapy (1 g azithromycin for chlamydia and $400 \mathrm{mg}$ cefixime for gonorrhea) at the school-based clinic, at the San Francisco STD clinic, or in the field from an STD Services staff member. Adolescents who had an STD diagnosed were encouraged strongly to refer their partners for treatment and to seek an additional STD examination and HIV testing.

Analyses, including chi-square tests, were conducted using Epi Info, version 6.04d (Centers for Disease Control and Prevention, Atlanta, GA).

\section{Results}

Fewer than $1 \%$ of parents did not consent to their adolescents' participation in the educational component of the program.

A total of 664 asymptomatic students were screened at the four schools: $2.1 \%$ (14) had chlamydia and $0.3 \%$ (2) had gonorrhea. All students with chlamydial or gonococcal infection were treated within 24 hours of availability of test results. Forty-three percent of those screened (283/664) were female; $46 \%$ of the students at the four schools (combined) were female.

The proportion of students infected with chlamydia or gonorrhea varied substantially by age and sex but did not vary significantly by high school after controlling for age and sex. Among the 283 females screened, 3.9\% (11) had chlamydia and $0.7 \%$ (2) had gonorrhea. The prevalence of chlamydia was $1.5 \%(1 / 65)$ among females younger than age 16 years and $4.6 \%$ (10/218) among females aged 16 years or older. No gonococcal infections were identified in females younger than age 17 years (0/196), and $1.6 \%$ of females aged 17 years or older $(2 / 150)$ were infected with gonorrhea. One female $(0.4 \%)$ had both chlamydia and gonorrhea.

Only $0.8 \%$ (3) of the males $(\mathrm{n}=381)$ had chlamydia and none had gonorrhea. There were no chlamydial infections in males younger than 17 years of age, and only $1.6 \%$ of males aged 17 or more years (3/185) were infected.

The efficiency (number of persons screened per STD Service staff hour) and completeness (proportion of total students screened) varied substantially, depending on the screening setting and the total student body (Table 1). The most efficient screening method was in a health fair setting (school B). However, on a large campus this method still screened only a small proportion of the student body. Conversely, at the campus with a small student body where entire classes were released for screening, screening was most complete, but least efficient (school D).

\section{Discussion}

STD education and screening was both feasible and acceptable in San Francisco high schools. We found that it 
was not possible to develop a standard method to perform screening at each of the schools, because the selected schools were highly variable in size, available space, and resources (only one school in San Francisco had a health clinic in 1999). The efficiency and completeness of screening varied substantially by school, largely because of the size of the school and the venue where we were able to perform the screening.

A moderate proportion of females screened in San Francisco high schools had chlamydia, especially those 16 or more years of age. The prevalence of infection was substantially lower in younger persons, especially younger males. The lower prevalence of infections in males was consistent with New Orleans high school data ${ }^{4,5}$ and 1999 youth detention intake screening data from San Francisco, which showed that $10.2 \%$ of females $(68 / 670)$ and $2.8 \%$ of males (44/1556) had chlamydial infection. ${ }^{7}$

This difference in prevalence by sex most likely reflects physiological differences (the immature cervix of young females is an enhanced target for Chlamydia) ${ }^{8}$ and sexual partnering patterns (females are on average several years younger than their male partners, and adult males involved with teenage girls have accounted for a higher rate of pregnancy and STDs than teen-teen relationships).9,10

We could not evaluate what proportion of screened students were sexually active. However, according to the Youth Risk Behavior Survey data collected during 1999, approximately $27 \%$ of San Francisco high school students reported ever having had sexual intercourse, which is lower than the national average of $50 \% .{ }^{11}$ Only $19 \%$ of students had had sex in the previous 3 months, and the proportion who ever had sex varied substantially by grade, from $20 \%$ of ninth-graders to $39 \%$ of twelfth-graders. ${ }^{11}$

The prevalence of chlamydia and gonorrhea may be higher among sexually active students than we observed among screened students, because younger students, in particular, may not have been sexually active. Approximately one quarter of students screened were younger than 16 years of age.

The observed prevalence in this pilot study was lower than in previous reports, ${ }^{2-6}$ which may reflect the overall lower prevalence of chlamydia in San Francisco than in Baltimore and New Orleans ${ }^{1}$ and suggests we may have screened some persons who were not sexually active. Because every epidemic is local, each county and state must look at its own chlamydia prevalence data to guide appro- priate screening recommendations and allocation of resources.

At a minimum, annual screening of sexually active adolescents by clinicians, regardless of their reason for visiting a clinician, is a critical component of an effective chlamydia control program. High school screening tailored to local epidemiology may be a critical adjunct to successful control of chlamydia, because it provides screening to the age group most likely to have undetected infections. Breaking the chain of chlamydial infection transmission is key to protecting the reproductive health of adolescents.

Based on local availability of resources for STD screening in high schools, we recommend the following: (1) performing chlamydia screening over gonorrhea screening, (2) performing female screening over male screening, and (3) screening students aged 16 years or older (juniors and seniors) over younger students.

\section{References}

1. US Department of Health and Human Services, Division of STD Prevention. Sexually Transmitted Disease Surveillance 1998. Atlanta: Centers for Disease Control and Prevention, September 1999.

2. Burstein GR, Gaydos CA, Diener-West MD, Howell MR, Zenilman JM, Quinn TC. Incident Chlamydia trachomatis infections among inner-city adolescent females. JAMA 1998; 280:521-526.

3. Burstein GR, Waterfield G, Joffe A, Zenilman JM, Quinn TC, Gaydos CA. Screening for gonorrhea and chlamydia by DNA amplification in adolescents attending middle school health centers: opportunity for early intervention. Sex Transm Dis 1998; 25:395-402.

4. Cohen DA, Nsuami M, Etame RB, et al. A school-based chlamydia control program using DNA amplification technology. Pediatrics 1998; 101:E1.

5. Cohen DA, Nsuami M, Martin DH, Farley T. Repeated school-based screening for sexually transmitted diseases: a feasible strategy for reaching adolescents. Pediatrics 1999; 104:1281-1285.

6. Marazzo JM, White CL, Krekeler B, et al. Community-based urine screening for Chlamydia trachomatis with a ligase chain reaction assay. Ann Intern Med 1997; 127:796-803.

7. Sexually Transmitted Disease Prevention and Control Services. San Francisco Sexually Transmitted Disease Annual Summary, 1999. San Francisco: San Francisco Department of Public Health, November 2000 .

8. Stamm WE. Chlamydia trachomatis infections of the adult. In: Holmes KK, Sparling PF, Mardh P-A, et al., eds. Sexually Transmitted Diseases. 3rd ed. San Francisco: McGraw-Hill, 1999:407-422.

9. Males M, Chew KSY. The ages of fathers in California adolescent births, 1993. Am J Public Health 1996; 86:565-568.

10. Males M. Adult liaison in the "epidemic" of "teenage" birth, pregnancy and venereal disease. J Sex Res 1992; 29:525-545.

11. San Francisco Unified School District, School Health Programs Department. Youth Risk Behavior Survey Middle and High School Level Summary Report, 1999. San Francisco: San Francisco Unified School District, 2000 